\title{
Control de presión arterial en paciente doblemente trasplantado pluripatológico mediante servicios profesionales farmacéuticos asistenciales: protocolo 'ImpacHTA'
}

\author{
María José Sanz Orejas', Enrique Martínez Carretero²0 \\ 1. Licenciada en Farmacia. Farmacéutica adjunta en Farmacia A. Sanz Doblado. 2. Doctor en Farmacia. Profesor titular en la Facultad de Farmacia de la \\ Universidad de La Laguna (Santa Cruz de Tenerife).
}

\section{PALABRAS CLAVE}

Hipertensión arterial, ImpacHta, SEFAC EXPERT, adherencia

\section{ABREVIATURAS}

AMPA: automedida de presión arterial

HTA: hipertensión arterial

PA: presión arterial

PRM: problema relacionado con el medicamento

RNM: resultado negativo de la medicación

SEH-LELHA: Sociedad Española de Hipertensión-Liga española para la lucha contra la hipertensión arterial

\section{KEYWORDS}

High blood pressure, ImpacHta, SEFAC EXPERT, adherence

\begin{abstract}
PRESENTACIÓN
Varón de 46 años, $96,2 \mathrm{~kg}, 172 \mathrm{~cm}$, pluripatológico sometido a un trasplante renopancreático en 2013 por una diabetes mellitus tipo I no controlada en su adolescencia, acude a la farmacia a retirar su medicación habitual. Durante el servicio de dispensación se detecta un PRM (problema relacionado con el medicamento) de falta de adherencia terapéutica (1), pues el paciente, de los tres fármacos prescritos para el tratamiento de su hipertensión arterial (HTA), no toma el amlodipino $5 \mathrm{mg}$, ya que "no le resulta efectivo contra sus dolores de cabeza" (motivo por el que cree que su médico lo pautó) (tabla 1).

En ese momento se le propone realizar una medida aislada en farmacia comunitaria de su presión arterial, resultando unos valores superiores a los recomendados (140/90 mmHg según las guías de práctica clínica para dicha situación) (2).
\end{abstract}

\section{INTERVENCIÓN}

1ª intervención (01-07 de julio de 2017)

Propuesta de automedida de la presión arterial AMPA (siguiendo protocolo impacHTA), cumpliendo con la toma de dos de los tres tratamientos antihipertensivos prescritos: ramipril $5 \mathrm{mg}$ y furosemida $40 \mathrm{mg}$.

Una vez llevado a cabo el tratamiento de datos mediante el programa SEFAC e_XPERT, se obtuvo el correspondiente informe en el que se reflejaba el no control de la patología (medidas superiores a las estipuladas por las sociedades científicas para AMPA, 135/85 mmHg.) (2) (figura 1).

El presente artículo corresponde al trabajo ganador del "Premio Servicio Profesional ImpacHTA" del VIII Congreso Nacional de Farmacéuticos Comunitarios de SEFAC (Alicante 2018).

Recibido: 05/06/2020

Aceptado: 23/08/2021

Disponible online: 06/10/2021
Financiación: ninguna.

Conflicto de intereses: ninguno.

Cite este artículo como: Sanz MJ, Martínez E. Control de presión arterial en paciente doblemente trasplantado pluripatológico mediante servicios profesionales farmacéuticos asistenciales: protocolo 'ImpacHTA'. Farmacéuticos Comunitarios. 2021 Oct 06; 13 (4): 43-47. doi:10.33620/FC.2173-9218.(2021/Nol13).004.06

Correspondencia: Maria José Sanz Orejas (mariajo-sanz@hotmail.com).

ISSN 1885-8619 @SEFAC (Sociedad Española de Farmacia Clínica, Familiar y Comunitaria). Todos los derechos reservados. 
Tabla 1 Estado de situación al inicio del caso

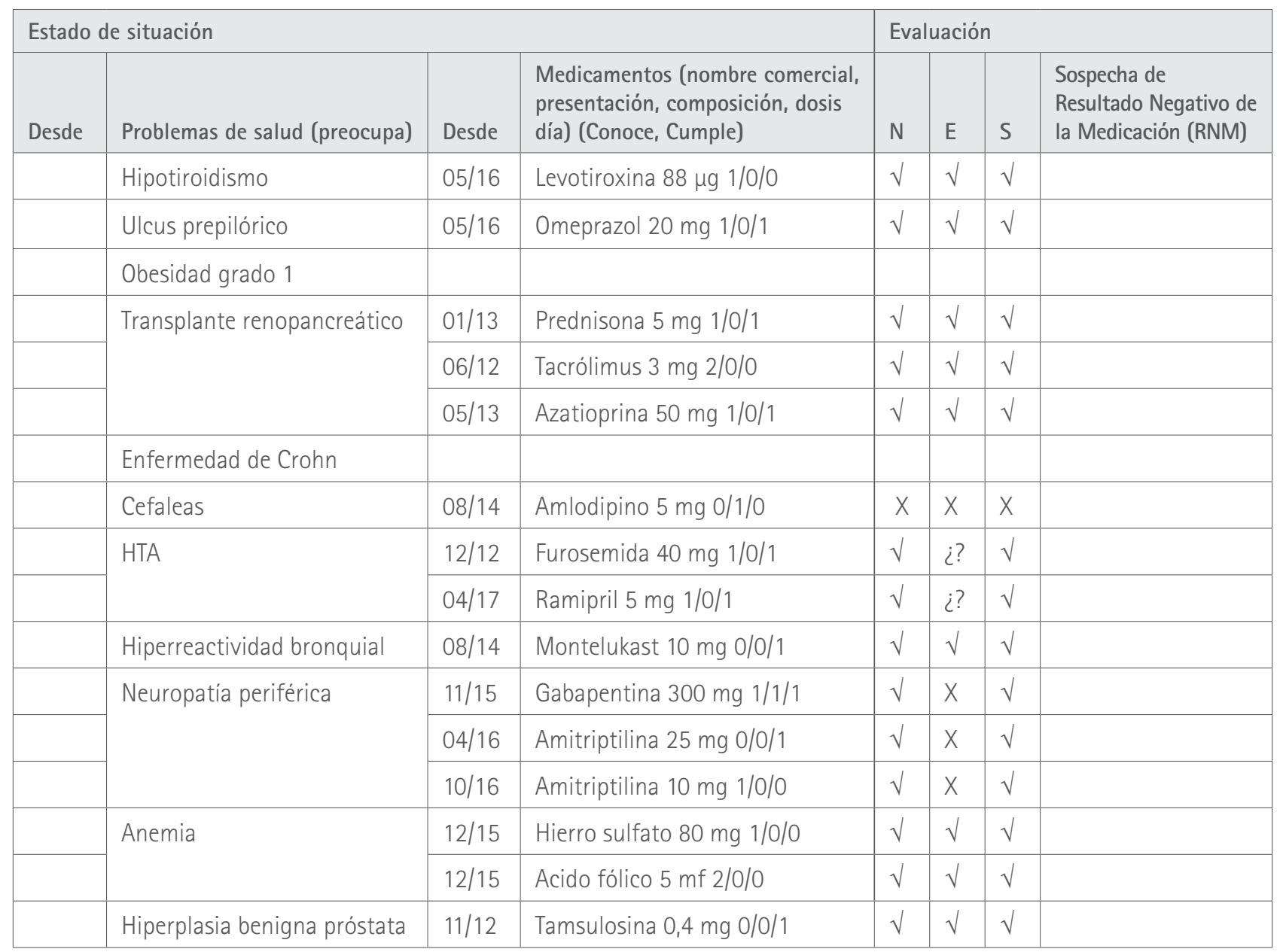

$X:$ problema; $\sqrt{ }=$ Correcto; $i ?=$ No se sabe.

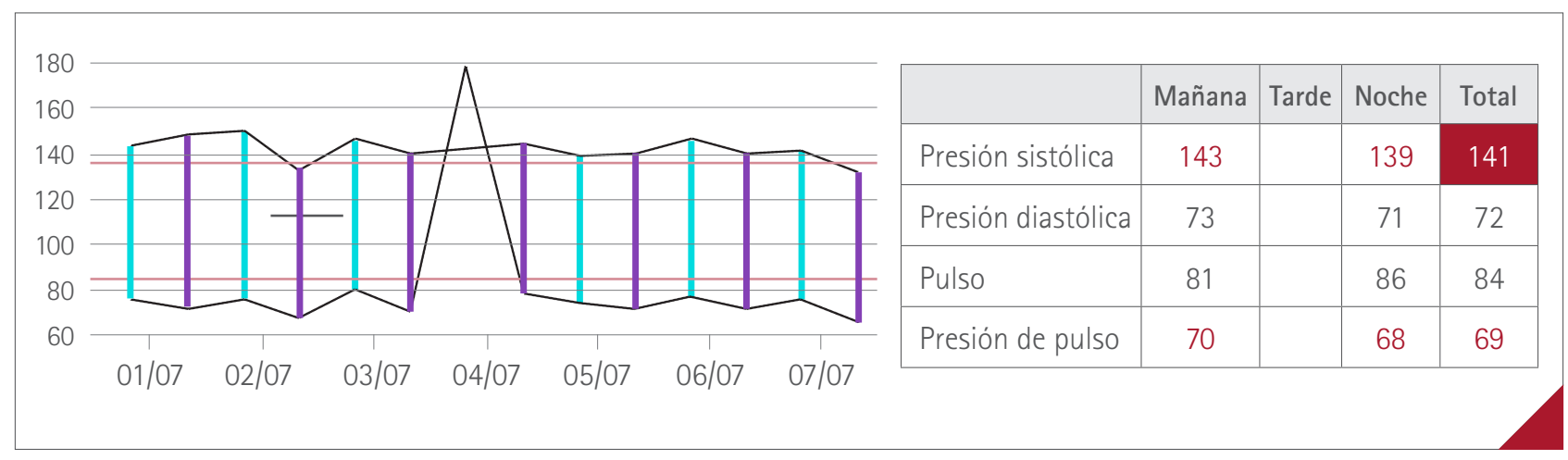

Figura 1 AMPA 1

\section{$2^{\text {a }}$ intervención (01-08 de agosto de 2017)}

Dado que los malos resultados obtenidos en el primer seguimiento de presión arterial se achacaron a una no adherencia terapéutica del paciente con respecto al amlodipino $5 \mathrm{mg}$., se le propone repetir el AMPA tras llevar un mes en tratamiento con los tres medicamentos prescritos (ramipril $5 \mathrm{mg}$ + furosemida $40 \mathrm{mg}$ + amlodipino $5 \mathrm{mg}$ ), recordándole la importancia de acompañar su tratamiento farmacológico de unos hábitos de vida saludables en consonancia con su condición de hipertenso (3).

A la vista de los valores obtenidos, donde la presión arterial (PA) no solo no mejoró, sino que empeoró, se decide involucrar al médico especialista a cargo de la tensión del paciente (figura 2). 


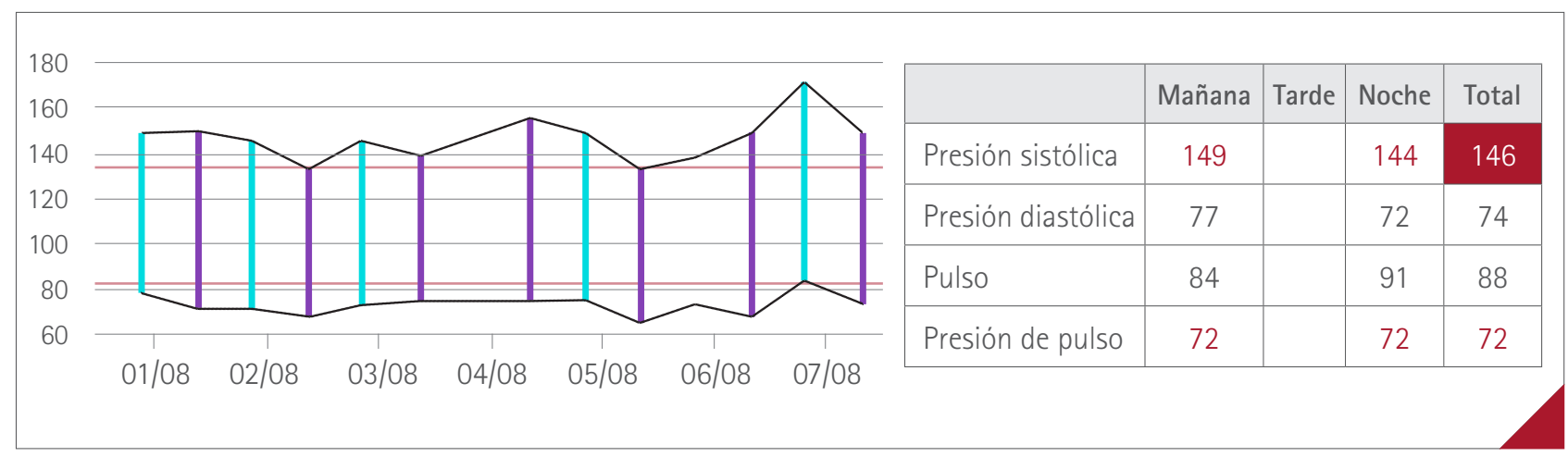

Figura 2 AMPA 2

\section{$3^{\text {a }}$ intervención (septiembre de 2017)}

A pesar de no padecer en la actualidad insuficiencia renal, se deriva al paciente a Nefrología, ya que es la especialidad a cargo del control de su tensión arterial desde que fue trasplantado de riñón. Se solicita que, atendiendo a los re- sultados del seguimiento, se reevalúe la terapia del mismo.

La nefróloga, aceptando nuestra intervención, pauta un cuarto medicamento antihipertensivo: doxazosina $4 \mathrm{mg}$, recomendándole al paciente que pasados unos meses repita el AMPA (tabla 2).

Tabla 2 Estado de situación tras la intervención 3

\begin{tabular}{|c|c|c|c|c|c|c|c|}
\hline \multicolumn{4}{|c|}{ Estado de situación } & \multicolumn{4}{|c|}{ Evaluación } \\
\hline \multirow[t]{20}{*}{ Desde } & Problemas de salud (preocupa) & Desde & $\begin{array}{l}\text { Medicamentos (nombre comercial, } \\
\text { presentación, composición, dosis } \\
\text { día) (Conoce, Cumple) }\end{array}$ & $\mathrm{N}$ & $\mathrm{E}$ & $\mathrm{S}$ & $\begin{array}{l}\text { Sospecha de } \\
\text { Resultado Negativo de } \\
\text { la Medicación (RNM) }\end{array}$ \\
\hline & Hipotiroidismo & $05 / 16$ & Levotiroxina $88 \mu \mathrm{g}$ 1/0/0 & $\sqrt{ }$ & $\sqrt{ }$ & $\sqrt{ }$ & \\
\hline & Ulcus prepilórica & $05 / 16$ & Omeprazol 20 mg 1/0/1 & $\sqrt{ }$ & $\sqrt{ }$ & $\sqrt{ }$ & \\
\hline & Obesidad grado 1 & & & & & & \\
\hline & \multirow[t]{2}{*}{ Trasplante renopancreático } & $01 / 13$ & Prednisona 5 mg 1/0/1 & $\sqrt{ }$ & $\sqrt{ }$ & ¿? & $\begin{array}{l}\text { Aumento de peso } \\
\text { HTA }\end{array}$ \\
\hline & & $06 / 12$ & Tacrólimus 3 mg 2/0/0 & $\sqrt{ }$ & $\sqrt{ }$ & ¿? & $\begin{array}{l}\text { HTA } \\
\text { Cefaleas } \\
\text { Anemia }\end{array}$ \\
\hline & & $05 / 13$ & Azatioprina 50 mg 1/0/1 & $\sqrt{ }$ & $\sqrt{ }$ & $\sqrt{ }$ & \\
\hline & Enfermedad de Crohn & & & & & & \\
\hline & \multirow[t]{4}{*}{ HTA } & $12 / 12$ & Furosemida $40 \mathrm{mg}$ 1/0/1 & $\sqrt{ }$ & $x$ & $\sqrt{ }$ & \\
\hline & & $04 / 17$ & Ramipril 5 mg 1/0/1 & $\sqrt{ }$ & $x$ & $\sqrt{ }$ & \\
\hline & & $08 / 14$ & Amlodipino $5 \mathrm{mg} \mathrm{0/1/0}$ & $\sqrt{ }$ & $x$ & $\sqrt{ }$ & \\
\hline & & $09 / 17$ & Doxazosina $4 \mathrm{mg} 1 / 1 / 1$ & $\sqrt{ }$ & $x$ & $\sqrt{ }$ & \\
\hline & Hiperreactividad bronquial & $08 / 14$ & Montelukast 10 mg 0/0/1 & $\sqrt{ }$ & $\sqrt{ }$ & $\sqrt{ }$ & \\
\hline & \multirow[t]{3}{*}{ Neuropatía periférica } & $11 / 15$ & Gabapentina 300 mg 1/1/1 & $\sqrt{ }$ & $x$ & $\sqrt{ }$ & \\
\hline & & $04 / 16$ & Amitriptilina 25 mg 0/0/1 & $\sqrt{ }$ & $x$ & $\sqrt{ }$ & \\
\hline & & $10 / 16$ & Amitriptilina $10 \mathrm{mg}$ 1/0/0 & $\sqrt{ }$ & $x$ & $\sqrt{ }$ & \\
\hline & Cefaleas & & & & & & \\
\hline & \multirow[t]{2}{*}{ Anemia } & $12 / 15$ & Hierro sulfato 80 mg 1/0/0 & $\sqrt{ }$ & $\sqrt{ }$ & $\sqrt{ }$ & \\
\hline & & $12 / 15$ & Ácido fólico 5 mg 2/0/0 & $\sqrt{ }$ & $\sqrt{ }$ & $\sqrt{ }$ & \\
\hline & Hiperplasia benigna de próstata & $11 / 12$ & Tamsulosina 0,4 mg 0/0/1 & $\sqrt{ }$ & $\sqrt{ }$ & $\sqrt{ }$ & \\
\hline
\end{tabular}

$X:$ problema; $V=$ Correcto; $i$ ? = No se sabe. 


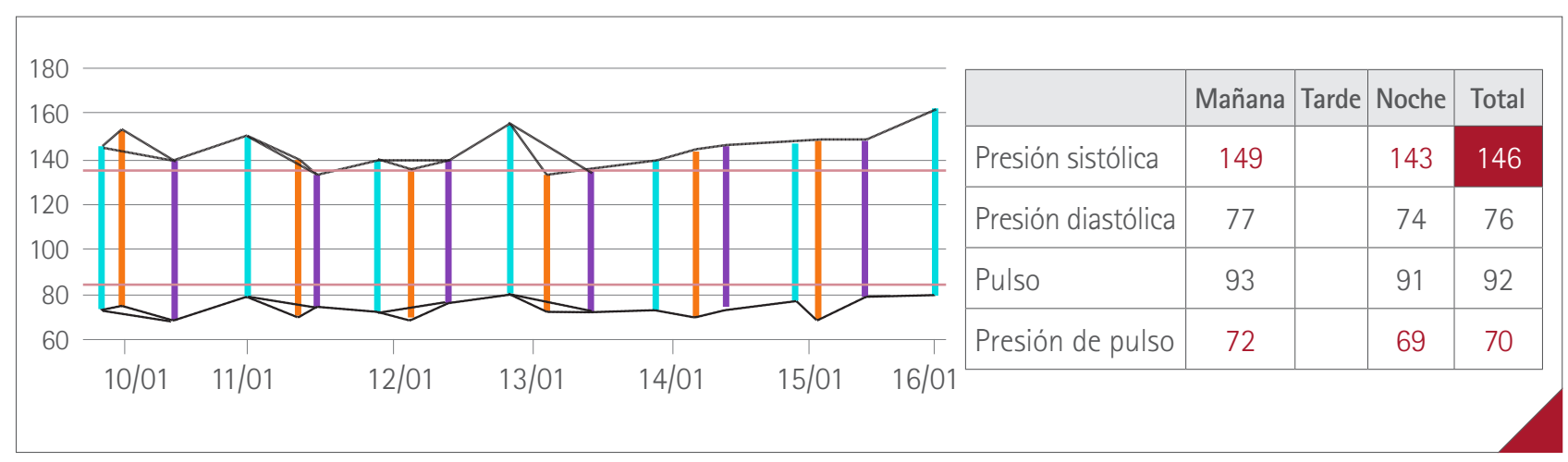

Figura 3 AMPA 3

Transcurridos cuatro meses desde que el paciente iniciara el tratamiento con doxazosina $4 \mathrm{mg}$, se repite el protocolo impacHTA para medidas domiciliarias (10-16 de enero de 2018), poniendo los resultados nuevamente de manifiesto la existencia de HTA grado I a pesar de la ampliación de tratamiento (figura 3).

Así las cosas, se plantea cuál o cuáles son los factores que pueden estar generando un fracaso de la terapia antihipertensiva.

La primera de las opciones evaluadas fue la posibilidad de encontrarnos ante una falta de adherencia farmacoterapéutica. A través de una Revisión del Uso de la Medicación (RUM) (4) por parte del paciente, se concluye que éste presenta un perfil de "crónico responsable" (colabora con el profesional sanitario, conoce su medicación, se interesa por su patología y toma sus tratamientos correctamente) (5), de modo que hubo que descartar dicho supuesto.

Otra de las opciones era la sospecha de que su HTA resistente se tratara de una reacción adversa propia de alguno de sus medicamentos o que fuera fruto de interacciones derivadas del arsenal terapéutico inmunosupresor que el paciente maneja debido a su condición de trasplantado. Dada la complejidad del mismo y la importancia vital de estos fármacos para el paciente, se obvió cualquier medida que pudiera involucrar este tipo de tratamientos.

Atendiendo a los hábitos de vida, nos encontramos ante un paciente con obesidad grado I $(I M C=32,5)$ y neuropatía invalidante que dificultan la realización de ejercicio, de modo que se consideró que no se obtendrian resultados con el impacto deseado a corto plazo. Es por ello que se decide actuar sobre el último factor que podría estar relacionado con el descontrol de su PA: la dieta; surgiendo la hipótesis de que se trate de un sujeto sodio-sensible (6).

\section{$4^{\text {a }}$ intervención (02-08 de marzo de 2018)}

Tras una entrevista exhaustiva en torno a los hábitos alimentarios del paciente, se propone una estrategia basada en la adherencia terapéutica más una dieta de evitación: durante los primeros tres días de la semana que dura el seguimiento AMPA, el paciente se alimentará como habitualmente viene haciéndolo y a partir del cuarto día no consumirá ninguno de los productos que le facilitamos en un listado (7).

\section{RESULTADO}

A partir del cuarto día se observa un punto de inflexión en el comportamiento de la presión arterial sistólica del paciente, pasando de encontrarse sus registros siempre por encima de los valores de referencia $(135 / 85 \mathrm{mmHg})$, a situarse en todo momento por debajo de los $135 \mathrm{mmHg}$. que marcan las guías de práctica clínica para AMPA. Se consiguió bajar sus valores de presión sistólica promedio de los $146 \mathrm{mmHg}$ del anterior AMPA, a $136 \mathrm{mmHg}$; es decir, se generó un descenso de $10 \mathrm{mmHg}$ evitando la ingesta de alimentos ricos en sodio (figura 4).

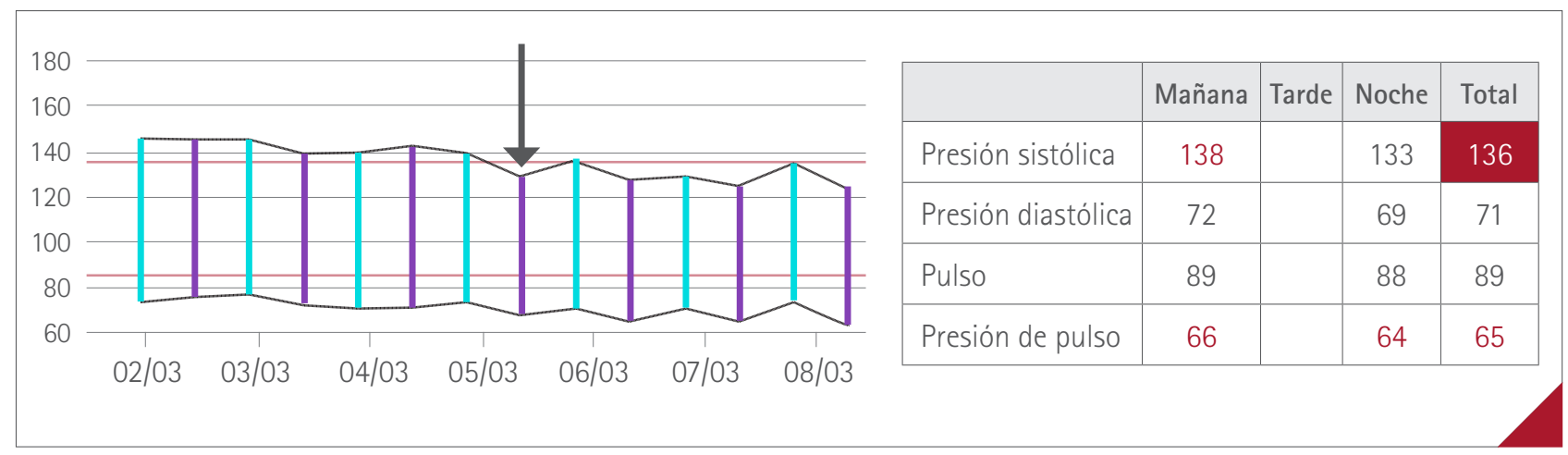

Figura 4 AMPA 4 


\section{CONCLUSIONES}

Los servicios profesionales farmacéuticos asistenciales resultan de gran utilidad para detectar posibles incidencias y potenciar/seguir la adherencia del paciente a sus tratamientos.

Ante resultados desconcertantes en los servicios de seguimiento de presión arterial, ha de plantearse la existencia de sodio-resistencia (hiperreacción al consumo de sal).

En ciertos casos no es suficiente con ajustes en el tratamiento farmacológico. Los pacientes precisan de asesoramiento sanitario personalizado y seguimiento por parte del farmacéutico comunitario, pues solo mediante la adherencia (entendiéndola como un equilibrio entre la terapia medicamentosa y la adopción de hábitos de vida saludables) (8) podremos optar al éxito terapéutico.

\section{REFERENCIAS BIBLIOGRÁFICAS}

1. AA.W. Guia práctica para los servicios de atención farmacéutica en farmacia comunitaria. Madrid: Editorial Consejo General de Colegios Oficiales de Farmacéuticos; 2010. Disponible en: https://www.portalfarma.com/inicio/serviciosprofesionales/forofarmaciacomunitaria/ Documents/2019-guia-practica-spfa.pdf

2. Divisón, JA, Penín O, Villasuso, B. Medida de presión arterial. Módulo I. En: Molinero A, Gómez Martínez JC, Iracheta Todó M, Martínez Pérez
SR, Martell Clarós N. Curso ImpacHTA. Madrid. Sociedad Española de Farmacia Comunitaria (SEFAC); 2014.

3. De la Figuera M, Sendra, J. Información personalizada del medicamentos y asesoramiento farmacéutico al paciente hipertenso y/o con riesgo vascular. En: Molinero A, Gómez Martínez JC, Iracheta Todó M, Martínez Pérez SR, Martell Clarós N. Curso ImpacHTA. Madrid: Sociedad Española de Farmacia Comunitaria (SEFAC); 2014.

4. Baixauli, VJ, Rua, FJ, Calle J, et al. Procedimiento normalizado de trabajo del servicio de revisión del uso de los medicamentos. Tema 1. En Curso REVISA. Madrid: Sociedad Española de Farmacia Comunitaria (SEFAC); 2017

5. Encuesta sobre adherencia terapéutica en España [Internet] Madrid. Farmaindustria. Último acceso 12/09/21. Disponible en: https://www. farmaindustria.es/web/documento/encuesta-adherencia-terapeutica-espanal

6. Coca A. Hipertensión esencial, sensibilidad a la sal y riesgo cardiovascular. Nefrología [revista en Internet]. 2004 [consultado en marzo 2018] Vol. 24. Núm. S1. Disponible en: https://revistanefrologia.com/es-hipertension-esencial-sensibilidad-sal-riesgo-articulo-X021169950402976X

7. Vocalía de farmacéuticos ejercientes en alimentación. Recomendaciones dietéticas: Protocolo Alimentario en Hipertensión. Colegio Oficial de Farmacéuticos de la región de Murcia y Sociedad murciana de Hipertensión y Riesgo cardiovascular. Disponible en: http://www.cofrm.com/web/Noticias.nsf/44943c3d3fa3d52ac1256b59003b3187/ 8dc045c0a9b2d92dc12574340033c131/\$FILE/FICHAS\%20HTA.pdf

8. Sabaté, E. Adherence to long-term therapies: evidence for action. Ginebra. World Health Organization; 2003. Disponible en: https://www. who.int/chp/knowledge/publications/adherence_introduction.pdf 